\title{
Half Mode Substrate Integrated Waveguide Cavity based RFID Chipless Tag
}

\author{
Soumaya Sakouhi ${ }^{1}$, Hedi Ragad ${ }^{2}$, Ali Gharsallah ${ }^{4}$ \\ Dept. of Physics, Faculty of Sciences of Tunis \\ University of Tunis El Manar, 2092 \\ Tunisia
}

\author{
Mohamed Latrach ${ }^{3}$ \\ RF-EMC Research Group, ESEO-IETR \\ CS90717-49107 Angers Cedex 2 \\ France
}

\begin{abstract}
This study presents the design of a compact Radio Frequency Identification chipless tag, with reduced size, important quality factor and improved results. The proposed prototype is based on Substrate Integrated Waveguide technology using the Half-Mode Substrate Integrated Waveguide technique to reduce the tag size by the half and preserve the same response. Further, the operating frequency band is from 5.5 to 8 $\mathrm{GHz}$ inside a compact surface of $1.3 \times 5.7 \mathrm{~cm}^{2}$. The frequency domain approach is adopted based on the frequency shift coding technique.
\end{abstract}

Keywords-Radio frequency identification (RFID); chipless tag; substrate integrated waveguide (SIW); ultra wide band (UWB)

\section{INTRODUCTION}

Wireless RF components and systems have been studied widely in recent years, and are constantly growing. Microwave literature is full of numerous enhancement tests in order to design high performance RF components with lower losses, higher power capacity and improved quality factor [1]. Substrate Integrated Waveguide technology has been emerged as a promising tool for the last research decade [2]. It has become extensively investigated and applied; and still proving day after day that it makes difference and improves structure such as mixers [3], oscillators [4], and Amplifiers [5]. Hence, it is constructed by using two rows of metalized cylinders or slots integrated in the dielectric substrate that electrically connect the two metal plates (patch and ground) [6]. Also, it is characterized by different shapes as circular, rectangular, and triangular structure. Recently, various applications have been already suggested for SIW technology. Mainly, the RFID chipless tag, our study focus, which is a promising alternative to develop a technology of identification more efficient than barcode and cheaper than conventional RFID, that uses tags equipped with Application-Specific Integrated Circuit (ASICS) associated to an antenna. The manufacturing and association tasks of each ASIC and antenna is expensive. This is the main reason for the limited utilization of conventional RFID to military tracking and some common transport application. As a result, RFID chipless tag has appeared since 2005 as an RF barcode which consists of 5 metallic strips giving 5 resonant peaks [7]. Besides, research focuses on improving chipless technology. In [8], an important data density is presented using 35 spiral resonators associated with cross polarized antennas coding 35 data bits. A 24-bit RFID tag is designed in [9], the tag operates in the UWB, and the presence or absence of resonant peak is the adopted technique to obtain data bits. The chipless tag in [10] uses three RF MEMS switch, giving 3-bit information over one period of the signal transmitted by an RFID reader. In [11], the tag encodes data using phase and frequency encoding technique. The proposed prototype in [12] is based on three split ring resonators, and it uses the geometrical angle relative to the reference ring resonator to encode data. SIW technology is applied in [13] using rectangular resonant cavity associated to an UWB antenna, as a chipless tag for space applications. Three tuning posts are used to shift frequency from 3.1 to $4.8 \mathrm{GHz}$.

From the research study, we can conclude that chipless technology is in intermediary phase, from the goal of improving the encoding capacity without being aware of the size and the application, towards a global reason which aims to combine the qualities of smart encoding technique, compact size, low losses, and reduced cost in industry. In this paper, a compact chipless tag based on SIW technology has an important quality factor is proposed. Its detailed behavior is presented theoretically and experimentally in Section 2. Also, it is tested and validated in Section 3.

\section{Presentation of the Half Mode Cavity based RFID CHIPLESS TAG BEHAVIOR}

\section{A. Half Mode Substrate Integrated Waveguide Cavity}

The most important characteristics that merit to be studied when designing a chipless tag are mainly, the encoding technique that ensures the validity of numerous encoding states (ID), the compact size that allows its application flexibility in several fields and the response performances include the efficiency, the stability and the quality factor. As known, SIW technology has distinctive advantages, particularly for the reason of combination of waveguide and planar technology features. It is a promising technology, which has been investigated and developed in the last decade [14]. It ensures a reduced size, low losses and an important quality factor. The propagation of electromagnetic waves in SIW-based structures has an important resemblance to its propagation in rectangular waveguide [15]. For this reason, several formulas were built in order to obtain the equivalent rectangular waveguide width. Hence, a formula can be estimated according to the geometrical parameters illustrated in Fig. 1, and using (1) below:

$$
a_{e q}=a_{S I W}-\frac{d}{0.95 p}^{2}
$$




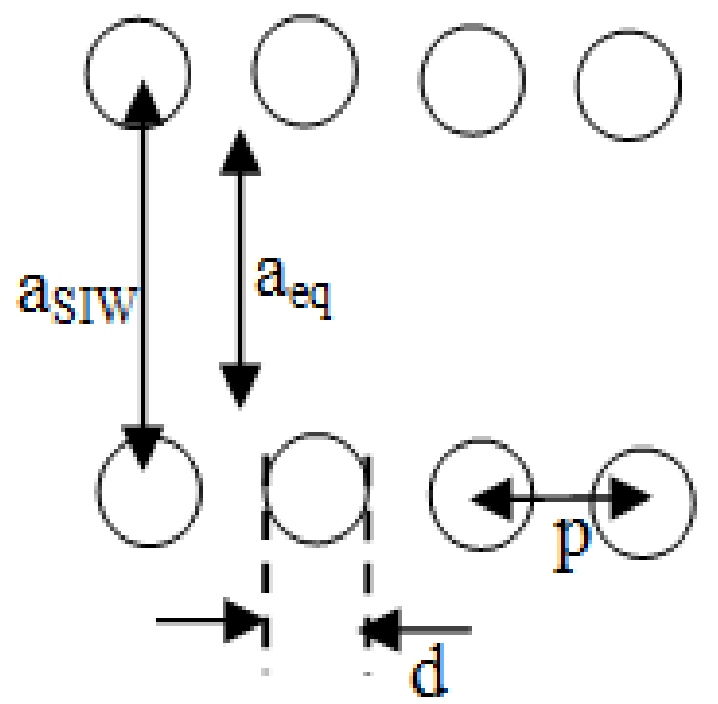

Fig. 1. Two metallic walls of metalized via in the SIW cavity.

Where, $\mathrm{a}_{\mathrm{eq}}$ is the waveguide width, $\mathrm{a}_{\text {sIw }}$ is the distance between two rows of metalized vias, $d$ is the diameter of each via, and $\mathrm{p}$ is the pitch which represents the spacing between two vias.

This section describes the use of SIW Technology aiming to develop chipless tag technology which consists of resonant cavity based on SIW technology associated to an UWB antenna characterized by an original shape and an UWB operation. Thus, the utilization of circular patch is chosen as an SIW cavity where a circular row of metalized via is drilled connecting the ground layer to the patch. The circular SIW cavity shape is studied. It has a width of $25 \mathrm{~mm}$ and a length of $30 \mathrm{~mm}$, as shown in Fig. 2(b) and Fig. 4(a). It has a radius $\mathrm{R}=$ $10.75 \mathrm{~mm}$, the metalized via radius is of $0.8 \mathrm{~mm}$, and a pitch of $1.89 \mathrm{~mm}$. Moreover, a 50-Ohm microstrip is designed as a feeding line which allows the measurements of the circular SIW cavity and permits connecting the cavity to the antenna in order to form the complete chipless tag.

The simulation of the Circular SIW (CSIW) cavity is built using the FR4 substrate, with a thickness of $0.7 \mathrm{~mm}$, a dielectric permittivity of 4.6 and loss tangent of 0.025 . The maximum electric fields are concentrated inside the cavity limited by the metalized via and symmetric along the $A B$ plane. The CSIW cavity is optimized, realized and measured. It operates in a sub band of the UWB from 5.5 to $8 \mathrm{GHz}$, giving a resonant peak at $5.825 \mathrm{GHz}$. The CSIW cavity prototype is shown in Fig. 2(b). Also, a good return loss characteristic is obtained at the operating frequency which exhibits a quality factor equal to 310. Therefore, the measurement is realized using the Agilent technology N5247A PNA-X network analyzer which covers the frequency range of $10 \mathrm{MHz}$ to 67 GHz.

Fig. 3 shows the distribution of the E-field in the circular cavity. The maximum electric fields are concentrated inside the cavity limited by the metalized via and symmetric along the AB plane.

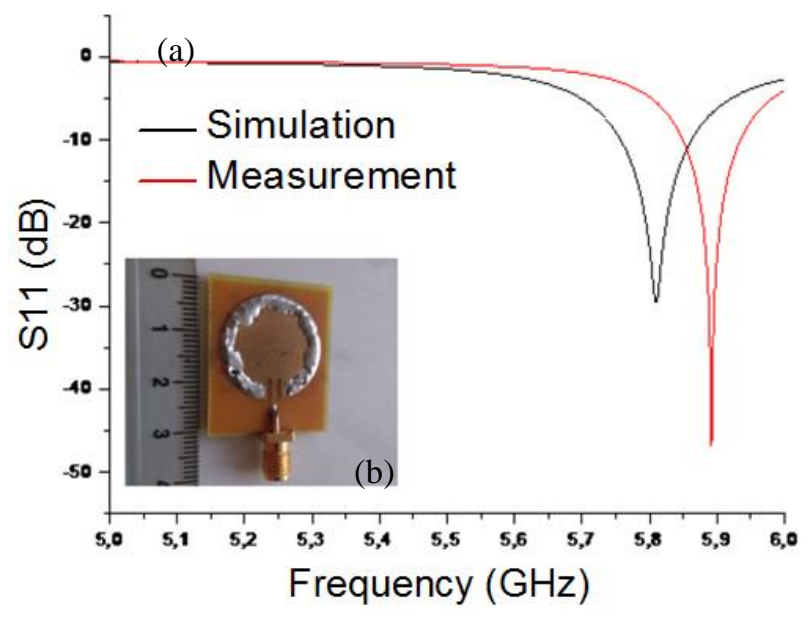

Fig. 2. (a) Simulated and measured $|S 11|$ of the Substrate Integrated Circular Cavity, (b) prototype photograph of circular SIW cavity.

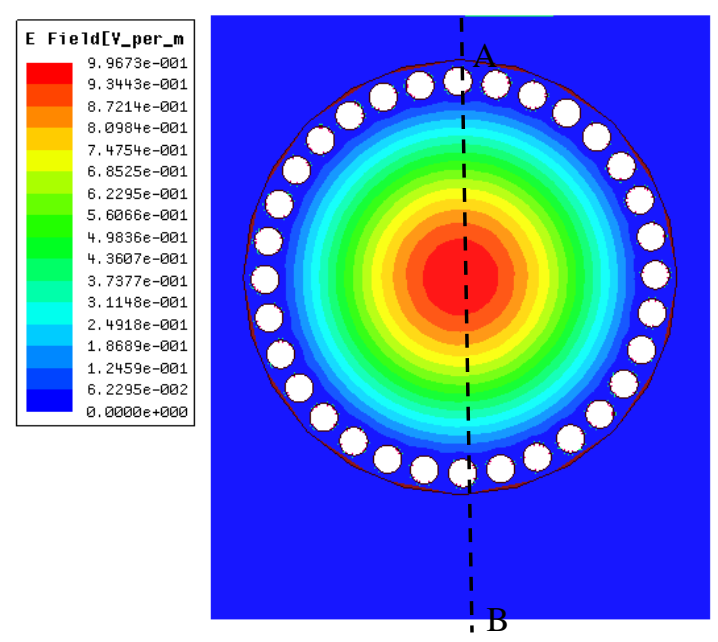

Fig. 3. Substrate integrated circular cavity.

The operating mode is the $\mathrm{TM}_{010}$. The corresponding resonant frequency of $\mathrm{TM}_{010}$ is obtained using (2) below:

$$
f_{010}=\frac{A_{010} c}{\pi R \sqrt{\varepsilon_{r} \mu_{r}}}
$$

Where $\mathrm{c}$ is the speed of light, $\mathrm{A}_{010}$ is the zero of the derivative of the Bessel function of $\mathrm{TM}_{010}, \mathrm{R}$ is the radius of the circular cavity, $\varepsilon_{\mathrm{r}}$ is the relative permittivity and $\mu_{\mathrm{r}}$ is the permeability of the substrate [16].
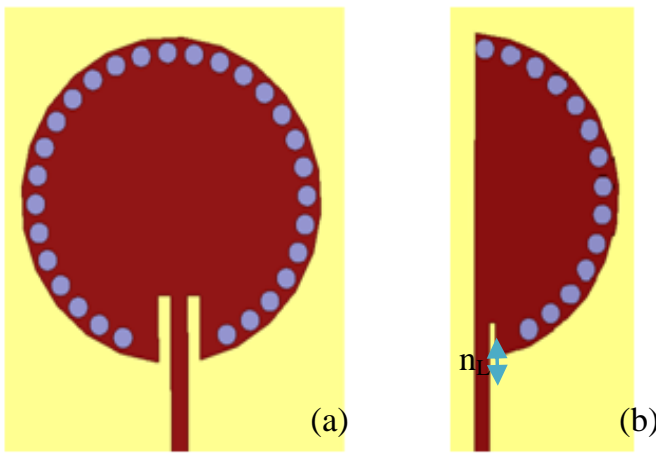

Fig. 4. The HMSIW technique: (a) the CSIW cavity, (b) the half-mode CSIW cavity. 
Therefore, by dividing the circular cavity in two halves along the $\mathrm{AB}$ plane, two half-mode cavities with same field distribution as the original circular cavity are obtained (see Fig. 5). the half-mode CSIW cavity is shown in Fig. 4(b), characterized by a notch length $\mathrm{n}_{\mathrm{L}}=2.2 \mathrm{~mm}$, and a notch width of $0.4 \mathrm{~mm}$. The spacement between the tag edge and the Halfmode cavity patch is of $2 \mathrm{~mm}$.

Fig. 6 above shows the Half-mode cavity responses of simulation and measurement. As already seen, the resonant peak of the configuration is the same of the whole structure at $5.825 \mathrm{GHz}$ with a slight difference in the bandwidth. The prototype is also shown in the same figure which is characterized by a reduced size compared to the complete circular cavity. The measured $|\mathrm{S} 11|$ is in good agreement with the simulated response giving a resonant peak at $5.825 \mathrm{GHz}$ (see Fig. 6(a)), which validates the technique of miniaturization. The size of the Half-mode circular SIW cavity is then $1 / 2$ of the circular SIW cavity with an identical resonant frequency which allows using the miniaturized half cavity as a main structure of our study.

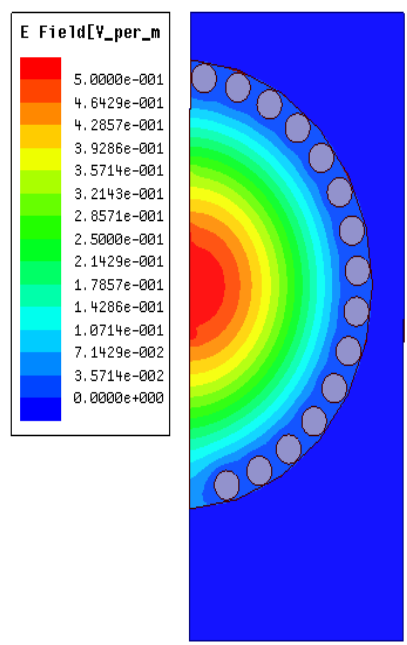

Fig. 5. The E-Field distribution in the HMSIW circular cavity.

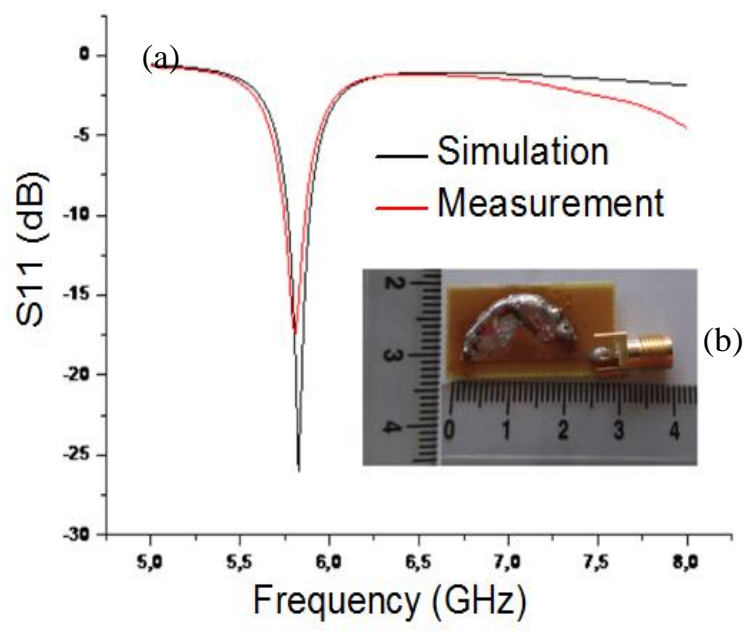

Fig. 6. (a) Simulated and measured $|\mathrm{S} 11|$ of the half-mode CSIW Cavity, (b) Prototype photograph.
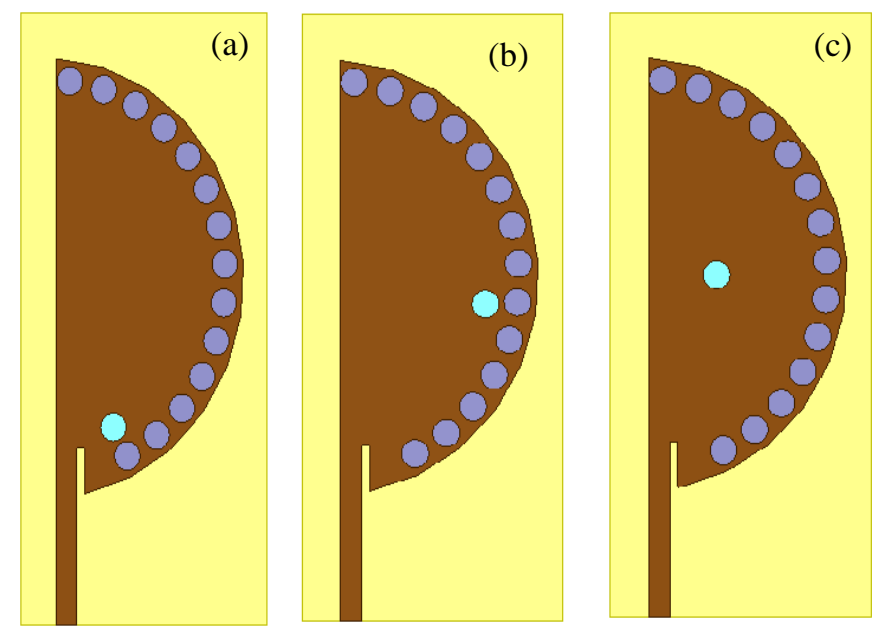

Fig. 7. The encoding technique using via holes and metalized via (a) first position, (b) second position, (c) third position.

Accordingly, the Half-Mode SIW circular cavity is the encoding area of our chipless tag. The frequency domain approach (FDA) is here adopted as an encoding approach using a simple frequency shifting technique which depends on the position and the nature of the via (air via or metalized via) inside the cavity substrate without influencing the tag size.

In fact, when an incident wave excites the Half-Mode cavity, it creates the fundamental resonant peak at $5.825 \mathrm{GHz}$ The concept in our actual study consists of demonstrate the encoding technique when adding via holes or metalized via in the substrate. Hence, the frequency domain approach is adopted using the frequency shifting technique. In Fig. 7, the manner of drilling via holes in the substrate is shown. It demonstrates that the first via hole drilled as given in Fig. 7(a), and respectively the second and the third position are shown in Fig. 7(b) and 7(c).

Hence, the via hole has a raduis of $0.7 \mathrm{~mm}$, and the HMSIW encoding surface proves that a frequency shift is realized afted drilling only one via hole.

Thus, the addition of the via hole in the first position gives a frequency shift to lower value (compared to the original cavity response at5.825 GHz) at $5.795 \mathrm{GHz}$ as shown in Fig. 8 . Changing to the second position ensures a new frequency value at $5.8 \mathrm{GHz}$. Further, changing to the third position gives a resonant frequency at $5.87 \mathrm{GHz}$. Consequently, the position changes of via holes shift the frequency to new values, mainly new encoding states. Each new position close to the maximum concentration of the E-field gives a maximum shift value that can be equal or great than $45 \mathrm{MHz}$. This is explained by the electric field distribution which is concentrated at the superior edge of the Half-Mode SIW cavity as explained in Fig. 5. Also, each modification in the substrate parameters close to the maximum field concentration brings a high frequency shifts.

Besides, the addition of metalized via with same radius of $0.7 \mathrm{~mm}$ influences the frequency to be shifted to higher values from a position to another. 


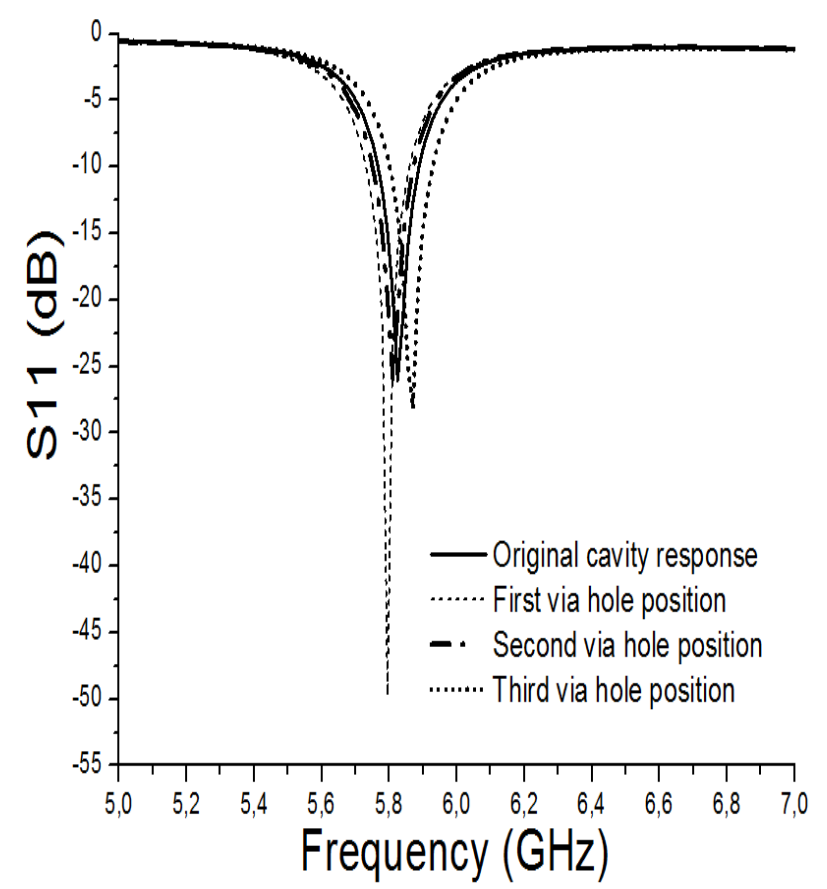

Fig. 8. Return loss responses of the HMSIW circular cavity after drilling via holes in three different positions.

Fig. 9 demonstrates the concept, and the metalized via placed at the first position gives a frequency shifts to 6.11 $\mathrm{GHz}$, which is an important shift of $285 \mathrm{MHz}$. Besides, the second position of metalized via permits a shift towards $6 \mathrm{GHz}$. The third position of metalized via is placed in a maximum Efield distribution (see Fig. 7(c)) gives an important shift to a new value at $7.8 \mathrm{GHz}$. This lead us to conclude that the metalized via gives important shifted values compared to via holes.

Accordingly, drilling via holes or metalized via leads to new encoding states where each new position in the Half-Mode SIW cavity gives a new frequency value.Further, adding $\mathrm{N}$ via holes or $\mathrm{N}$ metal via one after one in the substrate guides to $\mathrm{N}$ shifted frequency, mainly $\mathrm{N}$ encoding states.

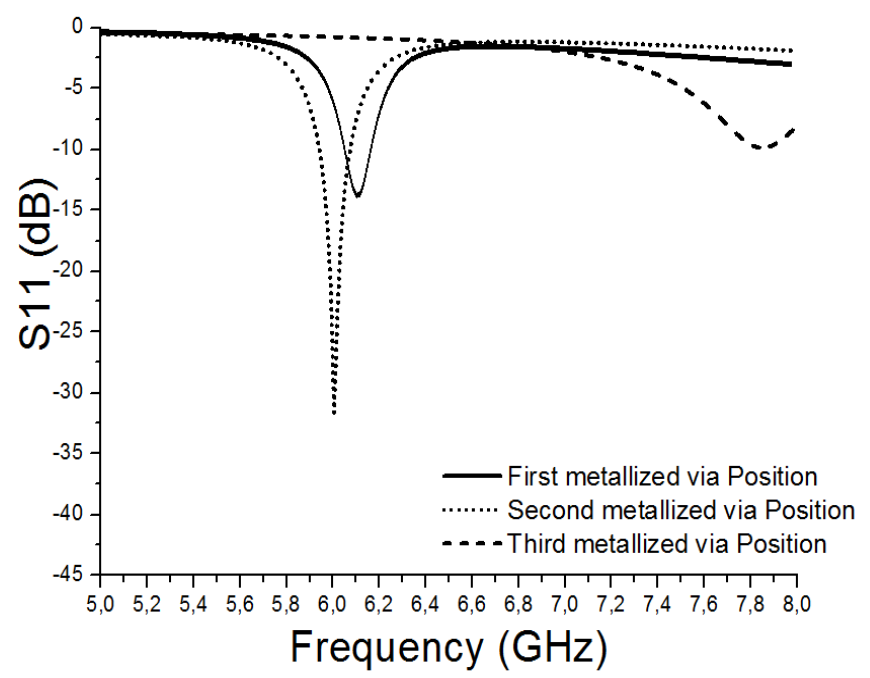

Fig. 9. The return loss responses of the HMSIW circular cavity after drilling metalized via in the substrate.

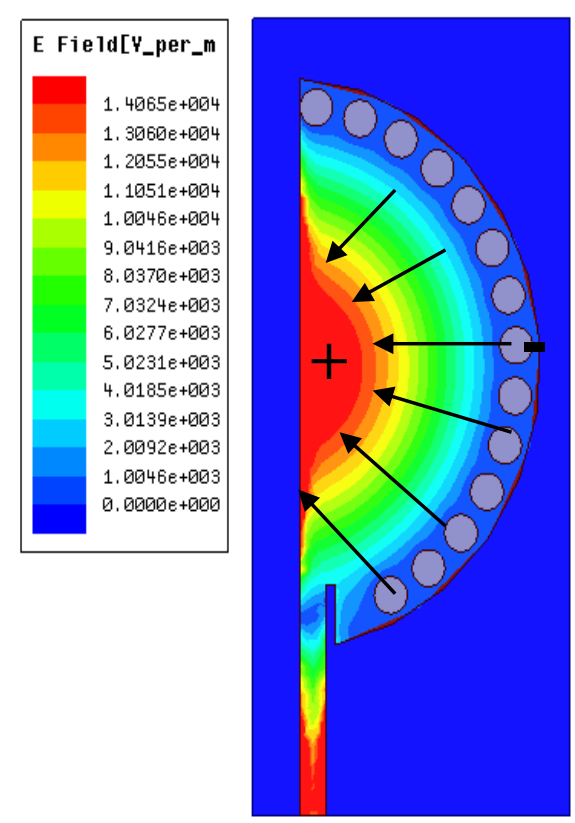

Fig. 10. The offset direction of the frequency values according to the E field distribution.

If we need to obtain close shifted values, we can drill via near to the EM wall created by the circular row of metallized via of the SIW technology. Also, we have the opportunity to obtain important shifts if metalized vias are drilled within the substrate in different positions. This shifts can reach and exceed $1 \mathrm{GHz}$ if the via is placed in the middle of the cavity where the $\mathrm{E}$ field is maximum. Fig. 10 shows the direction of the concentration of E-field, and mainly the frequency values increase gradually in the cavity each time vias are drilled closer to the E- field concentration.

\section{B. Ultra Wideband Crescent Shaped Antenna}

The second designed component is an essential part of the RFID tag which is the chipless tag antenna. It is the crescent shaped antenna that ensures the wideband operation.

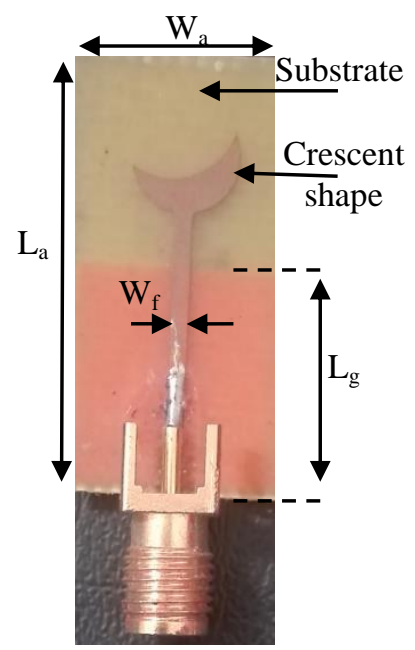

Fig. 11. Crescent UWB antenna. 


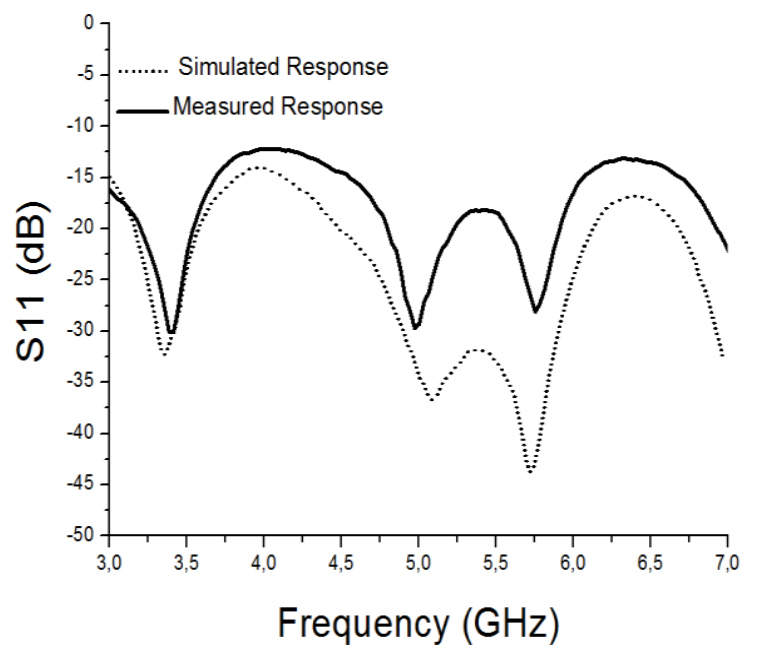

Fig. 12. Simulated and measured return loss of the crescent UWB antenna.

The crescent shaped antenna based chipless tag is characterized by a width of $\mathrm{W}_{\mathrm{a}}=13.5 \mathrm{~mm}$, and a length $\mathrm{L}_{\mathrm{a}}=27$ $\mathrm{mm}$. The ground antenna cover the lower part of the antenna without covering the radiating crescent element, it has a length $\mathrm{L}_{\mathrm{g}}=14 \mathrm{~mm}$. Fig. 11 shows the prototyped configuration.

The simulation results validates successfully the shape antenna choice and gives a gain of $1.3 \mathrm{dBi}$, and a well-adapted response from 3 to $7 \mathrm{GHz}$ as shown the return loss response in Fig. 12.

\section{Chipless TAG ASSOCIATION}

At this step, the two main components are ready to form the chipless tag based on SIW technology. Thus, the resonant HalfMode SIW cavity is associated with the UWB crescent antenna as shown in Fig. 13. The chipless tag is characterized by a compact size of $1.3 \times 5.7 \mathrm{~cm}^{2}$.

The simulation setup imitates the real operating conditions. The metallic rectangular waveguide WR-159 (40.386 x 20.193 $\mathrm{mm}^{2}$ ) with a mono-modal band from 4.90 to $7.05 \mathrm{GHz}$ was used as interrogator antenna to generate a broadband signal and to read the scattering radiation of the tag.

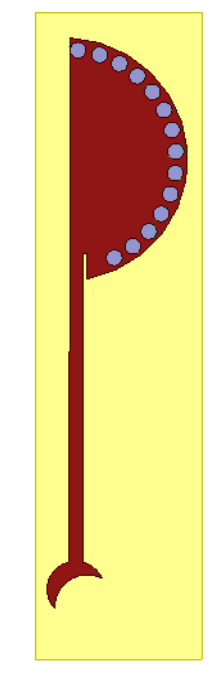

Fig. 13. The half-mode CSIW based tag.

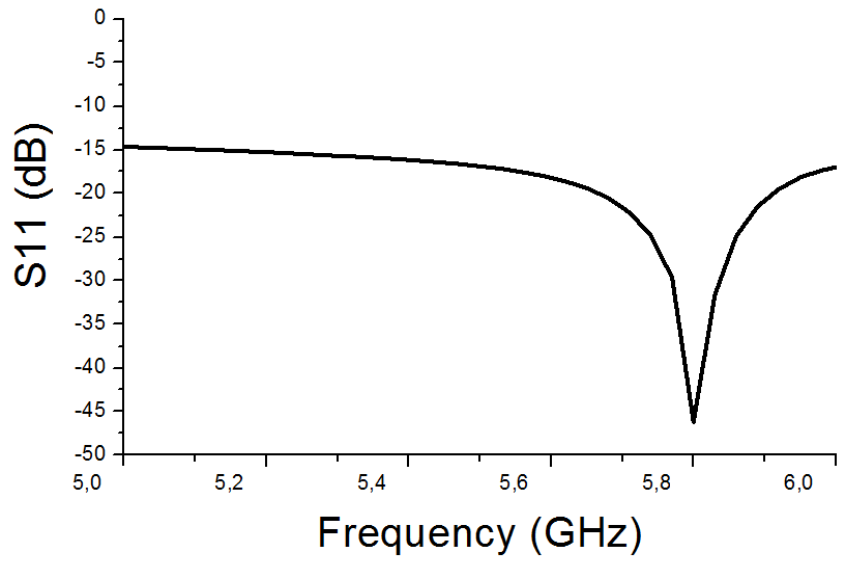

Fig. 14. Simulated tag response.

The chipless tag is placed under the waveguide separated by a distance of $1.5 \mathrm{~cm}$, with the center of the UWB antenna aligned with the center of the rectangular waveguide. In particular, in the simulated chipless system, the configuration of the cavity is chosen in order to resonate at $5.825 \mathrm{GHz}$.

The simulated response of the tag is shown in Fig. 14. It is therefore clear that the notch appeared in the frequency response is the signature of the tag under test, which validates our proposed design.

As evaluation, our proposed design uses an efficient technique of size reduction. Its feasibility has been proved by both considering the experimental measurements and the system simulation. It is characterized by an improved size compared to the square shaped chipless tag based on SIW technology proposed in [13]. It offers a numerous encoding states in a reduced surface using the frequency shifting technique from 5.5 to $8 \mathrm{GHz}$.

Hence, a significant improvement will be initiated in order to improve the encoding capacity in the same frequency band and with the same encoding approach using some promising techniques of SIW technology. Also, the FR4 substrate should be transferred to green materials in order to be easily inserted in credit cards or personal ID cards using the same HMSIWC based chipless tag.

\section{CONCLUSION}

A compact RFID chipless Tag based on SIW Technology has been designed in this paper using the technique of size reduction based on HMSIW technique. The HMSIW cavity and the UWB crescent shaped antenna based tag have theoretically and experimentally studied and validated on Ansoft HFSS. Using the frequency domain approach and referring to the modification of the effective permittivity of the substrate integrated resonator which gives a unique frequency signature. The tag can be easily modified by adding a via hole or metalized via in different position without touching the tag dimensions. The tag is able to obtain numerous encoding state, means high encoding efficiency using a reduced size cavity associated to a reduced size UWB antenna. The new tag has the advantage of having a high quality factor (310) as well as reduced size $\left(1.3 \times 5.7 \mathrm{~cm}^{2}\right)$. Thus, a research should be initiated 
to have the possibility to transfer the substrate to different types like paper, plastic and textile.

\section{REFERENCES}

[1] K. Finkenzeller, "RFID Handbook," 2nd edition, John Wiley \& Sons, 2003.

[2] M. Bozzi, A. Georgiadis, K. Wu, "Review of substrate-integrated waveguide circuits and antennas", IET Microw. Antennas Propag., 2011, Vol. 5, Iss. 8, pp. 909-920. DOI: 10.1049/iet-map.2010.0463.

[3] J.-X. Chen, W. Hong, Z.-C. Hao, H. Li, and K. Wu, "Development of a Low Cost Microwave Mixer Using a Broadband Substrate Integrated Waveguide (SIW) Coupler," IEEE Microwave and Wireless Components Letters, Vol. 16, No. 2, Feb. 2006. DOI: 10.1109/LMWC.2005.863199.

[4] Y. Cassivi and K. Wu, "Low Cost Microwave Oscillator Using Substrate Integrated Waveguide Cavity," IEEE Microwave and Wireless Components Letters, Vol. 13, No. 2, pp. 48 50, Feb. 2003. DOI: 10.1109/LMWC.2003.808720.

[5] K. W. Eccleston, "Corrugated Substrate Integrated Waveguide Distributed Amplifier", Proceedings of APMC 2012, Kaohsiung, Taiwan, Dec. 4-7, 2012. DOI: 10.1109/APMC.2012.6421604.

[6] C.Jin, R. Li, A. Alphones, X. Bao, "Quarter-Mode Substrate Integrated Waveguide and Its Application to Antennas Design", IEEE Transactions On Antennas And Propagation, VOL. 61, NO. 6, JUNE 2013. DOI: 10.1109/TAP.2013.2250238.

[7] I. Jalaly and I.D. Robertson, "RF barcodes using multiple frequency bands", in IEEE MTT-S Microwave Symp. Dig. Long Beach CA, June 2005, pp.139-141. DOI: 10.1109/MWSYM.2005.1516542.
[8] S. Preradovic, I. Balbin, N. C. Karmakar, "Multiresonator based chipless RFID system for low-cost item tracking", IEEE Transactions on Microwave Theory and Techniques, vol. 57, no. 5, pp:1411-1419, May 2009.

[9] R Reza, M. Majid, "Complex-natural-resonance-based design of chipless RFID tag for high-density data", IEEE Trans. Antennas Propag., 2014, 62, pp. 653- 656.

[10] A. Attaran, R. Rashidzadeh, R. Muscedere, "Chipless RFID tag using RF MEMS switch". Electronics Letters, 2014, vol. 50, no. 23, p. 1720 1722. DOI: 10.1049/el.2014.3075.

[11] A .Vena, E. Perret, S. Tedjini, "Chipless RFID tag using hybrid coding technique", IEEE Tran. Microw. Theory Tech., 2011, 59, pp. 33563364.

[12] A. Vena, E. Perret, S. Tedjini, "A compact chipless RFID tag using polarization diversity for encoding and sensing", IEEE Int. Conf. on RFID 2012, Orlando, USA, April 2012, pp. 191-197.

[13] S. Moscato, R. Moro, S. Sakouhi, M. Bozzi,, et al. „Chipless RFID for space applications. In Proceedings of the IEEE International Conference on Wireless for Space and Extreme Environments WISEE 2014. Noordwijk (The Neterlands), October 2014. DOI: 10.1109/WiSEE.2014.6973075.

[14] K. Wu, D. Deslandes, and Y. Cassivi, "The substrate integrated circuits-A new concept for high-frequency electronics and optoelectronics," in Proc. TELSIKS, Oct. 2003, pp. 3-5.

[15] M. Bozzi, A. Georgiadis, K. Wu, "Review of Substrate Integrated Waveguide (SIW) Circuits and Antennas," IET Microwaves, Antennas and Propagation, Vol. 5, No. 8, pp. 909920, June 2011.

[16] C. A. Balanis, Antenna Theory Analysis and Design, 3rd ed. New York, NY, USA: Wiley, 2005. 